\title{
Hecke algebras and hypergeometric functions
}

\author{
G.J. Heckman
}

Katholieke Universiteit Nijmegen, Department of Mathematics, Toernooiveld, 6525 ED Nijmegen, The Netherlands

\section{Contents}

1. Introduction

2. The Bernstein-Zelevinskii basis for the affine Hecke algebra versus the van der Lek-Looijenga presentation of the extended Artin group

3. Representation theory of affine Hecke algebras

4. The Schwarz problem

5. The classification of algebraic hypergeometric functions for root systems of type ADE

\section{§1. Introduction}

In the theory of special functions a frequently occurring theme is that of deformation of parameters. For example, the Jacobi polynomial $P_{n}^{(\alpha, \beta)}$ of degree $n$ with parameters $(\alpha, \beta)$ occurs as a zonal spherical harmonic on the projective spaces $\mathbf{P}^{k}(\mathbf{R}), \quad \mathbf{P}^{k}(\mathbf{C}), \quad \mathbf{P}^{k}(\mathbf{H})$ and $\mathbf{P}^{2}(\mathbf{O})$ for $(\alpha, \beta)=\left(\frac{1}{2} m_{1}+\frac{1}{2} m_{2}-\frac{1}{2}, \frac{1}{2} m_{2}-\frac{1}{2}\right)$ with $\left(m_{1}, m_{2}\right)=(k-1,0),(2 k-2,1),(4 k-4,3)$ and $(8,7)$ respectively. The restriction on the degree $n$ to be a nonnegative integer can be removed by going to the dual (in the sense of É. Cartan) hyperbolic space, and the discrete parameter $n$ gets replaced by a continuous spectral parameter. However the restriction on the parameters $(\alpha, \beta)$ as above is somewhat peculiar. Although slight extensions are possible one does get the feeling that Jacobi functions appear "in nature" only for a restricted set of rational parameters $(\alpha, \beta)$. Nevertheless general properties such as special values, growth behaviour and the differential equation go through for complex parameters $(\alpha, \beta)$. Moreover the harmonic analysis of the Jacobi functions as motivated by their interpretation of spherical harmonics goes through for real parameters $(\alpha, \beta)$ with $\alpha, \beta>-1$.

A similar phenomenon is going on for higher rank symmetric spaces. A large part of spherical function theory on a Riemannian symmetric space can be generalized, after restriction to a maximal split torus, to the case where the root multiplicities are allowed to be arbitrary real or complex parameters $[\mathrm{H} 0, \mathrm{H} 1, \mathrm{O} 1$, 
$\mathrm{O} 2, \mathrm{O} 3]$. We refer to these more general functions as hypergeometric functions associated with a root system. Having no longer the structure theory of symmetric spaces as a powerful tool available one has to look for more elementary methods, mainly from topology, algebraic geometry and complex analysis in several variables.

In [H1] a construction of these hypergeometric functions was given (at least for generic parameters) based on Deligne's solution of the Riemann monodromy problem in several variables [D]. This construction reduces to the construction of certain representations of the fundamental group of the complement of the global discriminant. This fundamental group has a presentation, due to van der Lek-Looijenga [vdL1, vdL2], which is called the extended Artin group. However it turns out that the monodromy representations of this extended Artin group all come from representations of the associated affine Hecke algebra. In Section 2 we explain this connection, and point out the analogy between the van der Lek-Looijenga presentation of the extended Artin group and the Bernstein-Zelevinskii basis for the affine Hecke algebra. In Section 3 we develop some (elementary) representation theory of affine Hecke algebras from which the required monodromy representations of the extended Artin group (for generic parameters) are obtained. Originally the existence of the monodromy representations of the extended Artin group was a consequence of rank two reduction and case by case differential algebraic calculations partly done on a computer [O1]. As an application of the results of Section 3 we give in Section 4 a solution to the Schwarz problem for which parameters the hypergeometric function is an algebraic function of its variables. For root systems of type ADE we work out the explicit parameter values in Section 5.

After this paper was written I received the preprint "Affine Hecke algebras and their graded version" by G. Lusztig, which has some overlap with Sections 2 and 3 of this paper.

I would like to thank the referee for useful comments.

\section{§2. The Bernstein-Zelevinskii basis for the affine Hecke algebra versus the van der Lek-Looijenga presentation of the extended Artin group}

Let $R \subset \mathfrak{a}^{*}$ be a reduced irreducible root system of rank $n$ with $\mathfrak{a}^{*}=\operatorname{Hom}(\mathfrak{a}, \mathbf{R})$ the real dual of a Euclidean vector space $\mathfrak{a}$ of dimension $n$. Let $\alpha^{\vee} \in \mathfrak{a}$ be the coroot of $\alpha \in R$ characterized by $\lambda\left(\alpha^{\vee}\right)=2(\lambda, \alpha) \cdot(\alpha, \alpha)^{-1} \forall \lambda \in \mathfrak{a}^{*}$, and $R^{\vee}=\left\{\alpha^{\vee} ; \alpha \in R\right\}$ the dual root system in a. Let $W \subset G L(\mathfrak{a})$ be the Weyl group generated by the reflections $r_{\alpha}(x)=x-\alpha(x) \alpha^{\vee}, \alpha \in R$. Fix a basis of simple roots $\left\{\alpha_{1}, \ldots, \alpha_{n}\right\}$ for $R$, and let $R_{+}$be the corresponding set of positive roots. Now $W$ has a presentation with generators the simple reflections $r_{1}, \ldots, r_{n}$ (we write $r_{i}$ for $r_{\alpha_{i}}$ ) and relations $\left(r_{i} r_{j}\right)^{m_{i j}}=1,1 \leqq i, j \leqq n$. Here $m_{i j} \in \mathbf{N}=\{1,2,3, \ldots\}$ are the Coxeter integers defined by $4 \cos ^{2}\left(\pi m_{i j}^{-1}\right)=\alpha_{i}\left(\alpha_{j}^{\vee}\right) \alpha_{j}\left(\alpha_{i}^{\vee}\right)$.

Let $Q^{\vee}=\mathbf{Z} \cdot R^{\vee} \subset$ a be the root lattice of $R^{\vee}$. The affine Weyl group $\tilde{W}$ is the group generated by $W$ and the translations $t_{x}$ over $x \in \mathbf{Q}^{\vee}$. It has a presentation with generators $r_{1}, \ldots, r_{n}, t_{1}, \ldots, t_{n}$ (we write $t_{i}$ for $\left.t_{\alpha_{i}^{\vee}}\right)$ and relations $\left(r_{i} r_{j}\right)^{m_{i j}}=1$, 
$t_{i} t_{j}=t_{j} t_{i}, r_{i} t_{j}=t_{j} t_{i}^{-n_{i j}} r_{i}, 1 \leqq i, j \leqq n$. Here $n_{i j} \in \mathbf{Z}$ are the Cartan integers defined by $n_{i j}=\alpha_{i}\left(\alpha_{j}^{v}\right)$.

The affine Weyl group $\tilde{W}$ has also a presentation as a Coxeter group with $(n+1)$ generators (with Coxeter diagram derived from the extended Dynkin diagram of $R$ ): Let $\alpha_{0}$ be the lowest root in $R$ (with respect to the partial ordering $\mu \leqq \lambda \Leftrightarrow \lambda-\mu=\sum_{1}^{n} k_{i} \alpha_{i}, k_{i} \in \mathbf{Z}_{+}=\{0,1,2, \ldots\}$ on $\left.\mathfrak{a}^{*}\right)$, and put $r_{0}=r_{\alpha_{0}} t_{\alpha_{0}^{\vee}}$ for the affine reflection in the hyperplane $\alpha_{0}(x)+1=0$. Then $\tilde{W}$ has a presentation with generators $r_{0}, r_{1}, \ldots, r_{n}$ and relations $\left(r_{i} r_{j}\right)^{m_{i j}}, 0 \leqq i, j \leqq n$ (the numbers $m_{0 i}=m_{i 0}$ are defined by the same formula as before). Let $l(w)$ denote the lengh of $w \in W$ relative to this presentation. For the definition of the Hecke algebra of a Coxeter group the standard reference is the exercises of Bourbaki [B, p. 55].

Definition 2.1. Let $c_{i} \in \mathbf{C}^{*}, i=0,1, \ldots, n$ be non zero complex parameters with $c_{i}=c_{j}$ if $r_{i}$ and $r_{j}$ are conjugated inside $\tilde{W}$ (if and only if the $i^{\text {th }}$ and $j^{\text {th }}$ node of the Coxeter diagram are connected by a chain of branches with odd marks). The affine Hecke algebra $\tilde{H}(c)$ of $\tilde{W}$ is a $\mathrm{C}$-vector space with basis $T_{w}, w \in \tilde{W}$ and multiplication rule

$$
\begin{array}{ll}
T_{r_{j}} T_{w}=T_{r_{j} w} & \text { if } l\left(r_{j} w\right)=l(w)+1, \\
T_{r_{j}} T_{w}=\left(1+c_{j}\right) T_{w}-c_{j} T_{r_{j} w} & \text { if } l\left(r_{j} w\right)=l(w)-1 .
\end{array}
$$

One can show that $\tilde{H}(c)$ as an algebra has a presentation with generators $T_{r_{j}}$, $j=0,1, \ldots, n$ and relations

$$
\begin{array}{rlrl}
\left(T_{r_{1}}-1\right)\left(T_{r_{j}}-c_{j}\right) & =0, & j=0,1, \ldots, n, \\
T_{r_{i}} T_{r_{j}} T_{r_{i}} \ldots & =T_{r_{j}} T_{r_{1}} T_{r_{j}}, \ldots, \quad 0 \leqq i \neq j \leqq n, \\
\text { and } m_{i j} \text { factors on both sides. }
\end{array}
$$

The Hecke algebra $\tilde{H}(c)$ is a deformation of the group algebra $\mathbf{C} \tilde{W}$ of $\tilde{W}$ corresponding to the case $c_{j}=-1, j=0,1, \ldots, n$. It is false that $l\left(t_{x} t_{y}\right)=l\left(t_{x}\right)+l\left(t_{y}\right)$ for all $x, y \in Q^{\vee}$. However $l\left(t_{x} t_{y}\right)=l\left(t_{x}\right)+l\left(t_{y}\right)$ does hold for all $x, y \in P_{+}^{\vee} \cap Q^{\vee}$ where $P_{+}^{\vee}=\left\{x \in \mathfrak{a}^{\vee} \alpha_{j}(x) \in \mathbf{Z}_{+}, j=1, \ldots, n\right\}$ is the set of dominant coweights. Indeed this is clear from the relation

$$
l\left(t_{x}\right)=2 h t(x), \quad x \in P_{+}^{\vee} \cap Q^{\vee} .
$$

Here $h t: Q^{\vee} \rightarrow \mathbf{Z}$ is the homomorphism defined by $h t\left(\alpha_{j}^{\vee}\right)=1, j=1, \ldots, n$ (or equivalently $\left.h t(x)=\rho(x), \rho=\frac{1}{2} \sum_{\alpha>0} \alpha\right)$ ). Hence $T_{t x} T_{t_{y}}=T_{t_{y}} T_{t_{x}}$ for all $x, y \in P_{+}^{\vee} \cap Q^{\vee}$. Now any $x \in Q^{\vee}$ can be written in the form $x=y-z$ with $y, z \in P_{+}^{\vee} \cap Q^{\vee}$, and the element

$$
T_{x}=T_{t_{y}} T_{t_{z}}^{-1} \in \tilde{H}(c)
$$

is well defined independently of the choice of $y, z \in P_{+}^{\vee} \cap Q^{\vee}$.

From the above it is clear that

$$
T_{x} T_{y}=T_{y} T_{x}=T_{x+y} \quad \text { for all } x, y \in Q^{\vee} .
$$

We use the notation $\mathrm{CQ}^{\vee}$ for the subalgebra of $\tilde{H}(c)$ generated by $T_{x}, x \in Q^{\vee}$. Indeed $\mathbf{C} Q^{\vee}$ is just the group algebra of $Q^{\vee}$. The following result goes back to Bernstein and Zelevinskii. 
Theorem 2.2. Besides (2.6) the relations

$$
\begin{gathered}
T_{r_{j}} T_{x}=T_{x} T_{r_{j}} \quad \text { if } \alpha_{j}(x)=0, \\
T_{r_{j}} T_{r_{j}(x)}=T_{x} T_{r_{j}}^{-1} \quad \text { if } \alpha_{j}(x)=1
\end{gathered}
$$

hold in the affine Hecke algebra $\tilde{H}(c)$.

For a proof of this theorem (in case all $c_{j}$ are the same, but this is not a serious restriction) we refer to [L1, p. 643] and [KL, p. 169].

Remark 2.3. We write $H(c)=H\left(c_{1}, \ldots, c_{n}\right)$ for the subalgebra of $\tilde{H}(c)=\tilde{H}\left(c_{0}, c_{1}, \ldots, c_{n}\right)$ generated by $T_{r_{j}}, j=1, \ldots, n$. Clearly $H(c)$ is the Hecke algebra of $\left(W,\left\{r_{1}, \ldots, r_{n}\right\}\right)$. We have

$$
\tilde{H}(c) \cong H(c) \otimes_{\mathrm{c}} \mathrm{C} Q^{\vee}
$$

as vector spaces, and the algebra structure on $\tilde{H}(c)$ can be recovered from the algebra structures on $H(c)$ and $C Q^{\vee}$ using (2.7), (2.8) and

$$
\left(T_{r_{0}}-1\right)\left(T_{r_{0}}-c_{0}\right)=0
$$

where $T_{-\alpha_{0}^{\vee}}=T_{r_{0}} T_{r_{0}}$ with $T_{-\alpha_{0}^{\vee}}=T_{\alpha_{1}^{1}}^{m_{1}} \ldots T_{\alpha_{n}^{\prime n}}^{m_{n}}$ if $\alpha_{0}^{\vee}+m_{1} \alpha_{1}^{\vee}+\ldots m_{n} \alpha_{n}^{\vee}=0$ and $T_{r_{\alpha_{0}}}=T_{r_{i_{1}}} \ldots T_{r_{i_{0}}}$ if $r_{\alpha_{0}}=r_{i_{1}} r_{i_{2}} \ldots r_{i_{p}}$ is a reduced expression.

Let $P=\left\{\lambda \in \mathbf{a}^{*} ; \lambda\left(\alpha^{\vee}\right) \in \mathbf{Z} \forall \alpha^{\vee} \in R^{\vee}\right\}$ be the weight lattice of $R$, and write $H$ for the complex torus with (rational) character lattice $P$. If $\mathfrak{h}=\mathbf{C} \otimes_{\mathbf{R}} \mathbf{a}$ denotes the complexification of $\mathfrak{a}$ then we have a short exact sequence

$$
0 \rightarrow 2 \pi i Q^{\vee} \rightarrow \mathfrak{h} \stackrel{\exp }{\longrightarrow} H \rightarrow 1
$$

For $\lambda \in P$ we write $h^{\lambda}$ for the corresponding character on $H$ (observe that $h^{\lambda}=e^{\lambda(\log h)}$ ). We denote by $\mathbf{Z}[H]$ the ring of Fourier polynomials on $H$ with integral coefficients. There is a natural action of $W$ on $\mathbf{Z}[H]$ and the invariants $\mathbf{Z}[H]^{W}$ are isomorphic to a polynomial algebra $\mathbf{Z}\left[z_{1}, \ldots, z_{n}\right]$ where

$$
z_{j}=\sum_{\mu \in \boldsymbol{W} \lambda_{j}} h^{\mu}, j=1, \ldots, n .
$$

Here $\left\{\lambda_{1}, \ldots, \lambda_{n}\right\}$ are the fundamental weights in $P_{+}$. Hence the quotient space $\tilde{W} \backslash \mathfrak{h}=W \backslash H=\mathbf{C}^{n}$. The Weyl denominator

$$
\Delta=h^{-\rho} \prod_{\alpha>0}\left(1-h^{\alpha}\right)
$$

transforms under $W$ according to the sign character. Hence $\Delta^{2}=D\left(z_{1}, \ldots, z_{n}\right)$ for some $D \in \mathbf{Z}\left[z_{1}, \ldots, z_{n}\right]$ which is called the (global) discriminant of $R$. The complement in $\mathbf{C}^{n}$ of the discriminant locus $D=0$ is the regular orbit space for $W$ on $H$, or equivalently for $\tilde{W}$ on $\mathfrak{h}$.

Definition 2.4. The Artin group $\tilde{G}$ belonging to $\left(\tilde{W},\left\{r_{0}, \ldots, r_{n}\right\}\right)$ is a group with generators $g_{0}, g_{1}, \ldots, g_{n}$ and relations

$$
g_{i} g_{j} g_{i} \ldots=g_{j} g_{i} g_{j} \ldots 0 \leqq i \neq j \leqq n, m_{i j} \text { factors on both sides }
$$


Theorem 2.5. The fundamental group of the complement in $\mathbf{C}^{n}$ of the discriminant locus $D=0$ has a presentation as the Artin group $\tilde{G}$ belonging to $\tilde{W}$.

This result is due to Nguyên Viêt Dung [N]. The corresponding result with $\tilde{W}$ replaced by $W$ was proved by Brieskorn [B], and the generalization to arbitrary Coxeter groups was given by van der Lek [vdL1, vdL2]. The following result was conjectured by Looijenga for type ADE, and proved by van der Lek in full generality.

Theorem 2.6. The Artin group $\tilde{G}$ belonging to $\tilde{W}$ has another presentation with generators $g_{1}, \ldots, g_{n}, l_{1}, \ldots, l_{n}$ and relations

$$
\begin{array}{ll}
g_{i} g_{j} g_{i} \ldots=g_{j} g_{i} g_{j} \ldots & 1 \leqq i \neq j \leqq n, m_{i j} \text { factors on both sides, } \\
l_{i} l_{j}=l_{j} l_{i} & 1 \leqq i, j \leqq n, \\
g_{i} l_{j}=l_{j} l_{i}^{k} g_{i} l_{i}^{-k} & 1 \leqq i \neq j \leqq n, n_{i j}=-2 k \text { even, } \\
g_{i} l_{j}=l_{j} l_{i}^{k+1} g_{i}^{-1} l_{i}^{-k} & 1 \leqq i \neq j \leqq n, n_{i j}=-(2 k+1) \text { odd, }
\end{array}
$$

and the element $g_{0}$ is given by $l_{1}^{m_{1}} l_{2}^{m_{2}} \ldots l_{n}^{m_{n}}=g_{0} g_{i_{1}} \ldots g_{i_{p}}$ with the same notation as in Remark 2.3. This presentation of $\tilde{G}$ is called the extended Artin group.

For an explicit description of the loops corresponding to the above generators we refer to [vdL1, vdL2]. We just remark that for the description given in Definition 2.4 one should take the base point in a fundamental alcove (for $\tilde{W}$ ) in $2 \pi i a$. For the description given in Theorem 2.6 one should take the base point in a fundamental chamber (for $W$ ) in $a$. The loops $l_{1}, \ldots, l_{n}$ generate the fundamental group of the torus $H$.

Remark 2.7. For $x \in Q^{\vee}$ of the form $x=k_{1} \alpha_{1}^{\vee}+\ldots+k_{n} \alpha_{n}^{\vee}$ we write $l_{x}=l_{1}^{k_{1}} \ldots l_{n}^{k_{n}} \in \tilde{G}$. Then it is easy to see that

$$
\begin{aligned}
l_{x} l_{y} & =l_{y} l_{x}=l_{x+y} & & \text { for all } x, y \in Q^{\vee}, \\
g_{j} l_{x} & =l_{x} g_{j} & & \text { if } \alpha_{j}(x)=0, \\
g_{j} l_{r_{j}}(x) & =l_{x} g_{j}^{-1} & & \text { for } \alpha_{j}(x)=1 .
\end{aligned}
$$

Observe that these relations are the analogues of the relations (2.6), (2.7), (2.8) in the affine Hecke algebra $\tilde{H}(c)$.

Proposition 2.8. If $\alpha_{j}\left(Q^{\vee}\right)=\mathbf{Z}$ then $l_{j} g_{j}^{-1}$ and $g_{j}$ are conjugate inside $\tilde{G}$. If $\alpha_{j}$ is a long simple root then $l_{j} g_{j}^{-1}$ and $g_{0}$ are conjugate inside $\tilde{G}$.

Proof. Suppose $\alpha_{j}(x)=1$ for some $x \in Q^{\vee}$. Using (2.21) we get

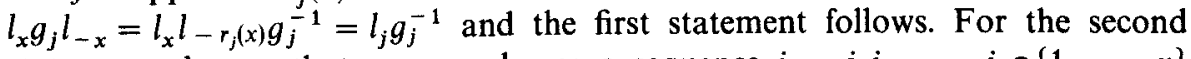
statement observe that we can choose a sequence $j_{1}=j, j_{2}, \ldots, j_{p} \in\{1, \ldots, n\}$ with

$$
\begin{aligned}
\beta_{k}^{\vee} & =\alpha_{j_{1}}^{\vee}+\ldots+\alpha_{j_{k}}^{\vee}, \\
\alpha_{j_{k+1}}\left(\beta_{k}^{\vee}\right) & =-1\left(\Leftrightarrow r_{j_{k+1}}\left(\beta_{k}^{\vee}\right)=\beta_{k+1}^{\vee}\right), \\
\beta_{p}^{\vee} & =-\alpha_{0}^{\vee}
\end{aligned}
$$


Now $r_{\beta_{k+1}}=r_{j_{k+1}} r_{\beta_{k}} r_{j_{k+1}}$ and $l\left(r_{\beta_{k+1}}\right)=l\left(r_{\beta_{k}}\right)+2$. Hence a reduced expression for $r_{\alpha_{0}}=r_{j_{p}} r_{j_{p-1}} \ldots r_{j_{2}} r_{j_{1}} r_{j_{2}} \ldots r_{j_{p}}$ and using (2.21) it is easily seen that

$$
g_{0}=l_{\beta_{p}^{\vee}} g_{j_{p}}^{-1} \ldots g_{j_{1}}^{-1} \ldots g_{j_{p}}^{-1}=\left(g_{j_{p}} \ldots g_{j_{2}}\right) l_{j} g_{j}^{-1}\left(g_{j_{p}} \ldots g_{j_{2}}\right)^{-1} \text {. Q.E.D. }
$$

Remark 2.9. We conclude that relation (2.10) is superfluous except for $R$ of type $C_{n}$ $\left(C_{1}=A_{1}, C_{2}=B_{2}\right), n \geqq 1$.

Proof. Indeed, if $R$ is not of type $C_{n}$ then each long simple root $\alpha_{j}$ satisfies $\alpha_{j}\left(Q^{\vee}\right)=Z$. Hence using Proposition 2.8 relation (2.10) is a consequence of the relations (2.6), (2.7), (2.8) and the quadratic relation $\left(T_{r_{j}}-1\right)\left(T_{r_{j}}-c_{j}\right)=0$. Q.E.D.

The system of hypergeometric differential equations is a system of differential equations on $C^{n}$ with regular singularities along the discriminant locus $D=0$ and at infinity [H0, H1, O1, O2]. Using (2.20) the monodromy representation could be computed explicitly by a rank one reduction.

Corollary 2.10. The monodromy representation of the hypergeometric differential equations, which is a priori a representation of the Artin group $\tilde{G}$, comes in fact from a representation of the affine Hecke algebra $\tilde{H}(c)$ for suitable $c=\left(c_{0}, c_{1}, \ldots, c_{n}\right)$.

Proof. By a rank one reduction it is clear that the generators $g_{1}, \ldots, g_{n}$ and $l_{1} g_{1}^{-1}, \ldots, l_{n} g_{n}^{-1}$ all satisfy quadratic relations in the monodromy representation. Hence the corollary follows from the second statement of Proposition 2.8. Q.E.D.

\section{§3. Representation theory of affine Hecke algebras}

Let $d_{j} \in \mathbf{C}^{*}, j=0,1, \ldots, n$ be complex parameters with $d_{i}=d_{j}$ if $r_{i}$ and $r_{j}$ are conjugated inside $\tilde{W}$, and assume that

$$
d_{j}^{2}=-c_{j}
$$

In this section we write $S \tilde{H}(d)$ for the affine Hecke algebra with generators $S_{r_{j}}, j=0,1, \ldots, n$ and quadratic relations

$$
\left(S_{r_{j}}-d_{j}\right)\left(S_{r_{j}}+d_{j}^{-1}\right)=0 \Leftrightarrow S_{r_{j}}=S_{r_{j}}^{-1}+\left(d_{j}-d_{j}^{-1}\right)
$$

together with the usual braid relations (2.3) with $T$ replaced by $S$. Observe that the correspondence $S_{r_{j}}=-d_{j}^{-1} T_{r_{j}}$ defines an isomorphism $\tilde{H}(c) \stackrel{\cong}{\rightarrow} S \tilde{H}(d)$ of algebras. For $x \in Q^{\vee}$ of the form $x=y-z$ with $y, z \in P_{+}^{\vee} \cap Q^{\vee}$ we keep the notation

$$
S_{x}=S_{t_{y}} S_{t_{z}}^{-1} \in S \tilde{H}(d)
$$

as in the previous section.

Proposition 3.1. Let $x \in Q^{\vee}$. For $\alpha_{j} \in R_{+}$a simple root with $\alpha_{j}\left(Q^{\vee}\right)=\mathrm{Z}$ we have

$$
S_{x} S_{r_{j}}=S_{r_{j}} S_{r_{j}(x)}+\left(d_{j}-d_{j}^{-1}\right) \frac{S_{x}-S_{r_{j}(x)}}{1-S_{-\alpha_{j}}}
$$


and with $\alpha_{j}\left(Q^{\vee}\right)=2 Z$ we have

$$
S_{x} S_{r_{j}}=S_{r_{j}} S_{r_{j}(x)}+\left\{\left(d_{j}-d_{j}^{-1}\right) S_{\alpha_{j}^{\vee}}+\left(d_{0}-d_{0}^{-1}\right)\right\} \frac{S_{x}-S_{r_{j}(x)}}{S_{\alpha_{i}^{\vee}}-S_{-\alpha_{j}}}
$$

Proof. Observe that the proof given by Lusztig of (2.7), (2.8) does not use the quadratic relations (2.2), and hence $(2.7),(2.8)$ also hold in $S \tilde{H}(d)$. In case $\alpha_{j}(x)=-1,0,1$ relation (3.4) follows immediately from (2.7), (2.8), and (3.4) holds for all $\alpha_{j}(x) \in \mathbf{Z}$ by an easy induction on $\left|\alpha_{j}(x)\right|$.

Now suppose $\alpha_{j}\left(Q^{\vee}\right)=2 Z$. By Proposition 2.8 we know that $S_{\alpha_{j}} S_{r_{j}}^{-1}$ and $S_{r_{0}}$ are conjugate inside $S \tilde{H}(d)$. Hence by (3.2)

$$
S_{\alpha_{j}^{\vee}} S_{r_{j}}=S_{r_{j}} S_{-\alpha_{j}^{\vee}}+\left(d_{j}-d_{j}^{-1}\right) S_{\alpha_{j}^{\vee}}+\left(d_{0}-d_{0}^{-1}\right),
$$

and for $x \in Q^{\vee}$ with $\alpha_{j}(x)=2$ we get from (2.7)

$$
S_{x} S_{r_{j}}=S_{r_{j}} S_{r_{j}(x)}+\left(d_{j}-d_{j}^{-1}\right) S_{x}+\left(d_{0}-d_{0}^{-1}\right) S_{x-\alpha_{j}^{v}} .
$$

Again (3.5) follows by induction on $\left|\alpha_{j}(x)\right|$. Q.E.D.

Remark 3.2. If $d_{0}=d_{j}$ then (3.5) becomes (3.4). As before we write $S H(d)=$ $S H\left(d_{1}, \ldots, d_{n}\right)$ for the subalgebra of $S \tilde{H}(d)$ generated by $S_{r_{j}}, j=1, \ldots, n$ and $\mathrm{CQ}^{\vee}$ for the (abelian) subalgebra of $S \tilde{H}(d)$ generated by $S_{x}, x \in Q^{\vee}$. From Proposition 3.1 it is obvious that (cf. Remark 2.3) multiplication in $S \tilde{H}(d)$ gives

$$
S \tilde{H}(d) \cong S H(d) \otimes_{\mathrm{c}} \mathrm{C} Q^{\vee}
$$

as vector spaces, and the algebra structure on $S \tilde{H}(d)$ can be recovered from the algebra structures on $S H(d)$ and $\mathrm{CQ}^{\vee}$ using (3.4) and (3.5).

Corollary 3.3. For $w=r_{i_{1}} \ldots r_{i_{p}} \in W$ a reduced expression we write $S_{w}=S_{r_{i_{1}}} \ldots S_{r_{i_{p}}} \in S H(d)$. Then we have for $x \in Q^{v}$

$$
S_{x} S_{w}-S_{w} S_{w^{-1}(x)} \in \sum_{u<w} S_{u} C^{v}
$$

where < denotes the Bruhat ordering on $W$.

Suppose $V$ is a finite dimensional $\mathrm{C}$-vector space equipped with the structure of a left $\widetilde{H}(d)$-module. By the Jordan decomposition we can write

$$
V=\underset{s \in \operatorname{Hom}\left(Q^{\vee}, \mathbf{C}^{*}\right)}{\bigoplus} V_{s}
$$

with

$$
V_{s}=\left\{v \in V ;\left(S_{x}-s(x)\right)^{p} v=0 \text { for } p \gg 0\right\}
$$

the generalized eigenspace. The set of all $s \in \operatorname{Hom}\left(Q^{\vee}, \mathbf{C}^{*}\right)$ with $V_{s} \neq 0$ are called the weights of $V$.

Definition 3.4. The left $S \tilde{H}(d)$-module $V(s, d)$ defined by

$$
V(s, d)=S \tilde{H}(d) / \sum_{z \in Q^{\vee}} S \tilde{H}(d)\left(S_{x}-s(x)\right)
$$

is called the induced $S \tilde{H}(d)$-module with cyclic weight $s \in \operatorname{Hom}\left(Q^{\vee}, \mathbf{C}^{*}\right)$. 
Remark 3.5. Clearly $V(s, d)$ is the universal left $S \tilde{H}(d)$-module generated by a vector of weight $s$. Using the isomorphism (3.6) it is clear that $V(s, d)$ as a vector space can be identified with $S H(d)$. In particular $\operatorname{dim} V(s, d))=|W|$ and the vectors $E_{w}=S_{w} \bmod \left(\sum_{x} S \tilde{H}(d)\left(S_{x}-s(x)\right) \in V(s, d), w \in W\right.$ are a basis for $V(s, d)$. By Corollary 3.3 it is clear that the weights of $V(s, d)$ are of the form $w(s), w \in W$. Indeed relative to the basis $E_{w}, w \in W$ of $V(s, d)$, partially ordered by the Bruhat ordering, the matrices of $S_{x}, x \in Q^{\vee}$ are upper triangular with $w(s)(x)$ on the diagonal.

The following result of Bernstein is now easily obtained (see [KL, p. 170]).

Proposition 3.6. The center $\tilde{Z}(d)$ of the generic (i.e. view $d_{j}$ as indeterminates rather than complex numbers) affine Hecke algebra $S \tilde{H}(d)$ is equal to the algebra $\left(\mathrm{CQ}^{\vee}\right)^{W}$.

Proof. Write $z \in \tilde{Z}(d)$ in the form $z=\sum z_{j}^{\prime} z_{j}^{\prime \prime}$ with $z_{j}^{\prime} \in S H(d)$ and $z_{j}^{\prime \prime} \in \mathbf{C} Q^{\vee}$. Applying $z$ to the cyclic vector $E_{1} \in V(s, d)$ and varying $s \in \operatorname{Hom}\left(Q^{\vee}, \mathbf{C}^{*}\right)$ shows that $z_{j}^{\prime}$ is a scalar. Hence $z \in \mathbf{C} Q^{\vee}$ and by Proposition 3.1 it is clear that $\left\{z \in \mathbf{C} Q^{\vee}\right.$; $\left.S_{r_{j}} z=z S_{r_{j}}, j=1, \ldots, n\right\}=\left(\mathbf{C} Q^{\vee}\right)^{W}$ for generic $d_{j}$. Q.E.D.

Proposition 3.7. For $j=1, \ldots, n$ with $\alpha_{j}\left(Q^{\vee}\right)=\mathbf{Z}$ the 2-dimensional representation generated by the matrix

and the matrices

$$
S_{r_{j}}=\left(\begin{array}{cc}
0 & 1 \\
1 & \left(d_{j}-d_{j}^{-1}\right)
\end{array}\right)
$$

$$
S_{x}=\left(\begin{array}{cc}
s(x) & \left(d_{j}-d_{j}^{-1}\right) \cdot \frac{s(x)-r_{j} s(x)}{1-s\left(-\alpha_{j}^{\vee}\right)} \\
0 & r_{j} s(x)
\end{array}\right), \quad x \in Q^{\vee}
$$

is irreducible if and only if

$$
\left(s\left(\alpha_{j}^{\vee}\right)-d_{j}^{2}\right)\left(s\left(-\alpha_{j}^{\vee}\right)-d_{j}^{2}\right) \neq 0 .
$$

For $j=1, \ldots, n$ with $\alpha_{j}\left(Q^{\vee}\right)=2 Z$ the 2-dimensional representation generated by the matrix (3.11) and the matrices

$$
S_{x}=\left(\begin{array}{cc}
s(x) & \left\{\left(d_{j}-d_{j}^{-1}\right) s\left(\alpha_{j}^{\vee}\right)+\left(d_{0}-d_{0}^{-1}\right)\right\} \cdot \frac{s(x)-r_{j} s(x)}{s\left(\alpha_{j}^{\vee}\right)-s\left(-\alpha_{j}^{\vee}\right)} \\
0 & r_{j} s(x)
\end{array}\right), \quad x \in Q^{\vee}
$$

is irreducible if and only if

$$
\left(s\left(\alpha_{j}^{\vee}\right)-d_{0} d_{j}\right)\left(s\left(-\alpha_{j}^{\vee}\right)-d_{0} d_{j}\right)\left(s\left(\alpha_{j}^{\vee}\right)+d_{0} d_{j}^{-1}\right)\left(s\left(-\alpha_{j}^{\vee}\right)+d_{0} d_{j}^{-1}\right) \neq 0 .
$$

Proof. If we write $E_{+}=E_{1}+d_{j} E_{r_{j}}$ and $E_{-}=E_{1}-d_{j}^{-1} E_{r_{j}}$ then $E_{+}$and $E_{-}$are the up to a constant unique eigenvectors of $S_{r_{j}}$ with eigenvalues $d_{j}$ and $-d_{j}^{-1}$ respectively. By a straightforward calculation we have for $\alpha_{j}\left(Q^{\vee}\right)=\mathbf{Z}$

$$
\begin{aligned}
& S_{x} E_{+}=r_{j} s(x) E_{+}+\frac{s(x)-r_{j} s(x)}{1-s\left(-\alpha_{j}^{\vee}\right)}\left(d_{j}^{2}-s\left(-\alpha_{j}^{\vee}\right)\right) E_{1} \\
& S_{x} E_{-}=r_{j} s(x) E_{-}+\frac{s(x)-r_{j} s(x)}{1-s\left(-\alpha_{j}^{\vee}\right)}\left(d_{j}^{-2}-s\left(-\alpha_{j}^{\vee}\right)\right) E_{1}
\end{aligned}
$$


and for $\alpha_{j}\left(Q^{\vee}\right)=2 Z$ we have

$$
\begin{gathered}
S_{x} E_{+}=r_{j} s(x) E_{+}+\frac{s(x)-r_{j} s(x)}{s\left(\alpha_{j}^{\vee}\right)-s\left(-\alpha_{j}^{\vee}\right)} \cdot\left\{d_{j}^{2} s\left(\alpha_{j}^{\vee}\right)-s\left(-\alpha_{j}^{\vee}\right)+d_{0} d_{j}-d_{0}^{-1} d_{j}\right\} E_{1} \\
S_{x} E_{-}=r_{j} s(x) E_{-}+\frac{s(x)-r_{j} s(x)}{s\left(\alpha_{j}^{\vee}\right)-s\left(-\alpha_{j}^{\vee}\right)} \cdot\left\{d_{j}^{-2} s\left(\alpha_{j}^{\vee}\right)-s\left(-\alpha_{j}^{\vee}\right)+d_{0} d_{j}^{-1}-d_{0}^{-1} d_{j}^{-1}\right\} E_{1}
\end{gathered}
$$

from which the proposition easily follows. Q.E.D.

Corollary 3.8. Let $R_{n r}=\{\lambda \in P ; \lambda \in R$ or $2 \lambda \in R\}$ be the possibly non reduced root system associated with $R$ (clearly $R_{n r}=R$ for $R$ of type $A(n \geqq 2), B(n \geqq 3), D(n \geqq 4)$, $E, F, G$ and $R_{n r}$ is of type $B C_{n}$ if $R$ is of type $\left.C_{n}(n \geqq 1)\right)$. For $\alpha \in R$ with $r_{\alpha}$ conjugate in $W$ to $r_{j}$ we write $d_{\alpha}=d_{j}$ if $\alpha \in R, \alpha\left(Q^{\vee}\right)=\mathbf{Z}$, and $d_{\alpha}=\left(d_{0} d_{j}\right)^{\frac{1}{2}}$ if $\alpha \in R, \alpha\left(Q^{\vee}\right)=2 Z$, and $d_{\frac{1}{2} \alpha}=\left(-d_{0}^{-1} d_{j}\right)^{\frac{1}{2}}$ if $\frac{1}{2} \alpha \in R_{n r} \backslash R$. If $s \in \operatorname{Hom}\left(Q^{\vee}, \mathbf{C}^{*}\right)$ is regular (i.e. $w_{1}(s) \neq w_{2}(s)$ for all $w_{1}, w_{2} \in W$ with $w_{1} \neq w_{2}$ ) and

$$
\prod_{\alpha \in R_{n r}}\left(s\left(\alpha^{v}\right)-d_{\alpha}^{2}\right) \neq 0
$$

then $V(s, d)$ is an irreducible left $S \tilde{H}(d)$-module.

Proof. The fact that $s \in \operatorname{Hom}\left(Q^{\vee}, \mathrm{C}^{*}\right)$ is regular together with Remark 3.5 implies the existence of a basis of simultaneous eigenvectors $F_{w}, w \in W$ in $V(s, d)$ for the operators $S_{x}, x \in Q^{\vee}$ such that the change of basis from $E_{w}$ to $F_{w}$ is given by a unipotent matrix (relative to the Bruhat ordering). Suppose $w \in W$ with $l\left(r_{j} w\right)=l(w)+1$. By Proposition 3.1 it follows that $\operatorname{span}_{\mathrm{C}}\left\langle F_{w}, F_{r_{j} w}\right\rangle$ is invariant under $S_{r,}$ and $S_{x}, x \in Q^{\vee}$. The corollary now follows from the previous proposition. Q.E.D.

Theorem 3.9. Suppose the system of hypergeometric partial differential equations (cf. [HO, Definition 2.13] and [O2, Theorem 3.6]) is put in standard Schrödinger form by conjugation with the weight function $\delta(k ; a)^{\frac{1}{2}}$ (cf. [HO, Proposition 2.2]). Then the corresponding monodromy representation of the Artin group $\tilde{G}$ coincides for generic parameters with the induced $S \tilde{H}(d)$-module $V(s, d)$ with cyclic weight $s \in \operatorname{Hom}\left(Q^{\vee}, C^{*}\right)$. The explicit correspondence between the parameters $(\lambda, k)$ and $(s, d)$ is given by

$$
s=e^{2 \pi i \lambda}
$$

and

$$
d_{\alpha}=e^{\pi i\left(k_{\ddagger a}+k_{\alpha}\right)}, \quad \alpha \in R_{n r}
$$

with the convention that $k_{\frac{1}{2} \alpha}=0$ if $\frac{1}{2} \alpha \notin R_{n r}$.

Proof. This is clear from Corollary 2.10, Remark 3.5, Corollary 3.8 and the formulas in Section 6 of [HO]. More precisely we find $d_{0}=e^{\pi i k_{a_{0}}}$ and

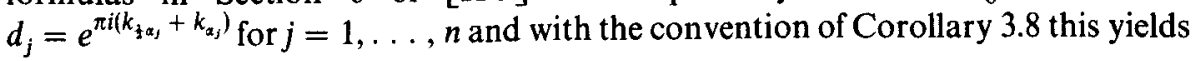
(3.18). Q.E.D.

Remark 3.10. Observe that for the application of the existence of hypergeometric functions and their shift operators the restriction in the above theorem to the case 
of generic parameters is irrelevant. Indeed, as shown by Opdam using a variation of Hartog's extension theorem the hypergeometric function $F(\lambda, k ; a)$ has an analytic continuation in the parameters $\lambda \in \mathfrak{h}^{*}$ and $k \in K$ with $\operatorname{Re}\left(k_{\alpha}\right) \geqq 0 \forall \alpha \in R_{n r}$. In turn this fact yields a proof of the existence of the commuting algebra of hypergeometric differential operators and their shift operators (see [O2, Section 2 and Section 3]). The essential point of the above theorem (although its proof is trivial) is therefore that it replaces the rather laborous calculations (partly done on a computer) in rank 2 as done in [O1].

\section{§4. The Schwarz problem}

In the notation of the previous section let

$$
\pi(s, d): \widetilde{G} \rightarrow G L(V(s, d))
$$

be the representation of the Artin group $\tilde{G}$ associated with the left $S \tilde{H}(d)$-module structure on $V(s, d)$. The Schwarz problem is the determination of those parameters $(\lambda, k)$ for which the hypergeometric function $F(\lambda, k ; z)$ is an algebraic function of its variables $z=\left(z_{1}, \ldots, z_{n}\right)$. Under the assumption that the monodromy representation is irreducible this problem is equivalent with the determination of those parameters $(s, d)$ for which the image of $\tilde{G}$ under the representation (4.1) is a finite group.

The method of solution of this question presented here is essentially the same as given in $[\mathrm{BH}]$ for the higher hypergeometric function ${ }_{n} F_{n-1}$.

Proposition 4.1. Relative to the basis $E_{w}, w \in W$ for $V(s, d)$ the representation (4.1) is defined over the ring

$$
\mathrm{Z}\left[d_{\alpha}, d_{\alpha}^{-1}, s\left(\alpha^{\vee}\right) ; \alpha \in R_{n r}\right]
$$

Proof. This is immediate from Proposition 3.1. Q.E.D.

Theorem 4.2. If the parameters $(s, d)$ satisfy (3.16) and the "hermitian condition"

$$
s=\vec{s}^{-1}, d_{\alpha}=\bar{d}_{\alpha}^{-1} \quad \forall \alpha \in R_{n r}
$$

(or equivalently $(\lambda, k)$ are real parameters), then there exists a non degenerate hermitian form $F=F(s, d)$ on $V(s, d)$ such that

$$
F\left(\pi(s, d)(g) v_{1}, \pi(s, d)(g) v_{2}\right)=F\left(v_{1}, v_{2}\right)
$$

for all $g \in \tilde{G}$, and all $v_{1}, v_{2} \in V(s, d)$.

Remark 4.3. Suppose $V$ is a finite dimensional vector space over $\mathbf{C}$, and $\pi: G \rightarrow G L(V)$ a representation of a group $G$. The existence of a bijective intertwining operator between $(\pi, V)$ and $\left(\bar{\pi}^{*}, \bar{V}^{*}\right)$ is equivalent with the existence of a $G$-invariant non degenerate sesquilinear pairing $S: V \times V \rightarrow \mathbf{C}$. Here $\left(\bar{\pi}^{*}, \bar{V}^{*}\right)$ is the anti dual representation defined by $\bar{\pi}^{*}(g)=\overline{\pi\left(g^{-1}\right)^{*}}$. Clearly

$$
F^{\prime}(v, w)=\frac{1}{2}\left(S(v, w)+\overline{S(w, v))} \quad \text { and } \quad F^{\prime \prime}(w, v)=\frac{1}{2 i}(S(v, w)-\overline{S(w, v))}\right.
$$


are both $G$-invariant hermitian forms with $\operatorname{Ker}\left(F^{\prime}\right) \cap \operatorname{Ker}\left(F^{\prime \prime}\right)=0$. Hence for $\lambda, \mu \in \mathbf{R}$ generic the hermitian form $F=\lambda F^{\prime}+\mu F^{\prime \prime}$ is $G$-invariant and non degenerate.

Proposition 4.4. We have the equivalence of representations

$$
\overline{\pi(s, d)^{*}} \cong \pi\left(\overline{w_{0} s^{-1}}, \bar{d}^{-1}\right) \text {. }
$$

In particular, if $s$ and $d$ are unitary then

$$
\overline{\pi(s, d)^{*}} \cong \pi\left(w_{0} s, d\right)
$$

Here $w_{0} \in W$ is the longest element.

Proof. If we write $E_{w}=E_{w}(s, d), w \in W$ for the basis of $V(s, d)$ as described in Remark 3.5 then it is easy to check using Proposition 3.7 that the equivalence (4.5) comes from

$$
E_{w}\left(\overline{w_{0} s^{-1}}, \bar{d}^{-1}\right)=\overline{E_{w w_{0}}(s, d)^{*}},
$$

where $\bar{E}_{w}^{*}, w \in W$ is the basis of $\overline{V(s, d)^{*}}$ dual to $E_{w}, w \in W$. Q.E.D.

Definition 4.5. Using Proposition 3.7 it is easy to check that the vector

$$
-\left(d_{j}-d_{j}^{-1}\right) E_{1}\left(r_{j} s, d\right)+\left(1-r_{j} s\left(-\alpha_{j}^{\vee}\right)\right) E_{r_{j}}\left(r_{j} s, d\right)
$$

in case $\alpha_{j}\left(Q^{\vee}\right)=\mathbf{Z}$, and the vector

$$
\begin{gathered}
-\left\{\left(d_{j}-d_{j}^{-1}\right) r_{j} s\left(\alpha_{j}^{\vee}\right)+\left(d_{0}-d_{0}^{-1}\right)\right\} E_{1}\left(r_{j} s, d\right)+ \\
\left\{r_{j} s\left(\alpha_{j}^{\vee}\right)-r_{j} s\left(-\alpha_{j}^{\vee}\right)\right\} E_{r_{j}}\left(r_{j} s, d\right)
\end{gathered}
$$

in case $\alpha_{j}\left(Q^{\vee}\right)=2 \mathrm{Z}$ is a simultaneous eigenvector in $V\left(r_{j} s, d\right)$ of weight $s$ for the operators $S_{x}, x \in Q^{\vee}$.

Hence there exists a unique intertwining operator of left $\tilde{H}(d)$-modules

$$
I_{j}: V(s, d) \rightarrow V\left(r_{j} s, d\right)
$$

sending the cyclic vector $E_{1}(s, d)$ to the vector (4.7) and (4.8) respectively.

Proposition 4.6. The composition

$$
V(s, d) \stackrel{I_{j}}{\rightarrow} V\left(r_{j} s, d\right) \stackrel{I_{j}}{\rightarrow} V(s, d)
$$

is given by multiplication with a scalar which is non zero if and only if the conditions (3.13) and (3.15) respectively are satisfied. In particular under conditions (3.13) and (3.15) respectively the operator $I_{j}: V(s, d) \rightarrow V\left(r_{j} s, d\right)$ is a bijection.

Proof. A straightforward computation. Q.E.D.

Proposition 4.7. For $w \in W$ with $w=r_{j_{1}} \ldots r_{j_{p}}$ a reduced expression the intertwining operator

$$
I_{w}=I_{j_{1}} \ldots I_{j_{p}}: V(s, d) \rightarrow V(w s, d)
$$

is well defined independently of the choice of the reduced expression. 
Proof. It is easily seen that with respect to the basis $E_{v}(w s, d), v \in W$ of $V(w s, d)$ the coefficient of $E_{w}(w s, d)$ in the vector $I_{w}\left(E_{1}(s, d)\right)$ is given by

$$
\prod_{\substack{\beta \in R_{+} \cap w^{-1} R_{-} \\ \beta\left(Q^{\vee}\right)=\mathbf{Z}}}\left(1-s\left(\beta^{\vee}\right)\right) \cdot \prod_{\substack{\beta \in R_{+} \sim w^{-1} R_{-} \\ \beta\left(Q^{\vee}\right)=2 Z}}\left(s\left(-\beta^{\vee}\right)-s\left(\beta^{\vee}\right)\right) .
$$

Hence for regular $s \in \operatorname{Hom}\left(Q^{\vee}, \mathbf{C}^{*}\right)$ the intertwining operator $I_{w}$ depends only on $w$, and not on the choice of the reduced expression. The proposition now follows because $I_{w}$ depends polynomially on $s$. Q.E.D.

Corollary 4.8. For $(s, d)$ parameters satisfying $(3.16)$ the intertwining operator

$$
I_{w_{0}}: V(s, d) \rightarrow V\left(w_{0} s, d\right)
$$

is a bijection.

The proof of Theorem 4.2 now follows from Remark 4.3, Proposition 4.4 and Corollary 4.8 .

Theorem 4.9. Suppose the parameters $(s, d)$ satisfy (3.16). Then the image under the representation (4.1) of the Artin group $\tilde{G}$ is a finite group if the parameters $(s, d)$ satisfy the condition

$$
s^{N}=1, d_{\alpha}^{N}=1 \quad \forall \alpha \in R_{n r}, \quad \text { some } N \in \mathbf{N}
$$

(or equivalently $(\lambda, k)$ are rational parameters), and for each $\sigma \in \operatorname{Gal}(K(s, d): \mathbf{Q})$ where

$$
K(s, d)=\mathbf{Q}\left(s\left(\alpha^{\vee}\right), d_{\alpha} ; \quad \alpha \in R_{n r}\right)
$$

the hermitian form $F\left(s^{\sigma}, d^{\sigma}\right)$ on $V\left(s^{\sigma}, d^{\sigma}\right)$ is definite.

Proof. By Proposition 4.1 the representation (4.1) is defined over the ring of integers (4.2) in the cyclotomic field $K(s, d)$. Now the diagonal embedding

$$
\pi(s, d)(\tilde{G}) \rightarrow \prod_{\sigma \in \operatorname{Gal}(K(s, d): \mathbf{Q})} \pi\left(s^{\sigma}, d^{\sigma}\right)(\tilde{G})
$$

is defined over $\mathbf{Z}$, and leaves invariant a positive definite hermitian form. Hence the image is a finite group. Q.E.D.

Corollary 4.10. The function $F(\lambda, k ; z)$ is an algebraic function of its variables $z=\left(z_{1}, \ldots, z_{n}\right)$ if the parameters $(\lambda, k) \in \mathfrak{h}^{*} \times K$ are rational and all rank one reductions of $F(\lambda, k ; z)$ (which are Gaussian hypergeometric functions) appear in the list of H.A. Schwarz.

Proof. In case the representation (4.1) is irreducible the invariant hermitian form on $V(s, d)$ is definite if and only if all rank one reductions of the hermitian form are definite. Indeed, for $s \in \operatorname{Hom}\left(Q^{\vee}, \mathbf{C}^{*}\right)$ regular for $W$ this is immediate, and the statement depends continuously on $s$ as long as (4.1) remains irreducible. The corollary now follows because the criterium of Theorem 4.9 holds as soon as it holds for all rank one reductions. Q.E.D. 


\section{\$5. The classification of algebraic hypergeometric functions for root systems of type ADE}

Definition 5.1. Two triples $(\lambda, \mu, v)$ and $\left(\lambda^{\prime}, \mu^{\prime}, v^{\prime}\right)$ in $\mathbf{R}^{3}$ are called contiguous if one is obtained from the other by applying finitely many operations of the form

$$
\begin{aligned}
& (\lambda, \mu, v) \mapsto(-\lambda, \mu, v) \text { or }(\lambda,-\mu, v) \text { or }(\lambda, \mu,-v), \\
& (\lambda, \mu, v) \mapsto(\lambda+2, \mu, v) \text { or }(\lambda, \mu+2, v) \text { or }(\lambda, \mu, v+2), \\
& (\lambda, \mu, v) \mapsto(\lambda, 1-\mu, 1-v) \text { or }(1-\lambda, \mu, 1-v) \text { or }(1-\lambda, 1-\mu, v)
\end{aligned}
$$

Definition 5.2. A triple $(\lambda, \mu, v) \in \mathbf{R}^{3}$ is called reduced if and only if $0 \leqq \lambda, \mu, v$ and $\lambda+\mu, \lambda+v, \mu+v \leqq 1$.

Proposition 5.3. Any class of contiguous triples in $\mathbf{R}^{3}$ contains a unique reduced triple.

Proof. If we enlarge the group generated by the transformations (5.1), (5.2), (5.3) with the symmetric group $S_{3}$ of permutations of $\lambda, \mu, v$ then the statement is that the fundamental alcove $\{0 \leqq \lambda \leqq \mu \leqq \nu, \mu+v \leqq 1\}$ is a fundamental domain for the action of the affine Weyl group of type $B_{3}$. Q.E.D.

The Gaussian hypergeometric function $F(\alpha, \beta, \gamma ; z)$ has exponent differences $\lambda=1-\gamma, \mu=\gamma-\alpha-\beta, v=\alpha-\beta$ around $z=0,1, \infty$ respectively. The following result is due to H.A. Schwarz [S].

Theorem 5.4. (H.A. Schwarz). The hypergeometric function $F(\alpha, \beta, \gamma ; z)$ has an irreducible monodromy group and is an algebraic function of its variable $z$ if and only if the contiguity class of exponent differences

$$
\lambda=1-\gamma, \quad \mu=\gamma-\alpha-\beta, \quad v=\alpha-\beta
$$

contains as reduced triple and up to permutation one of the following 15 triples:

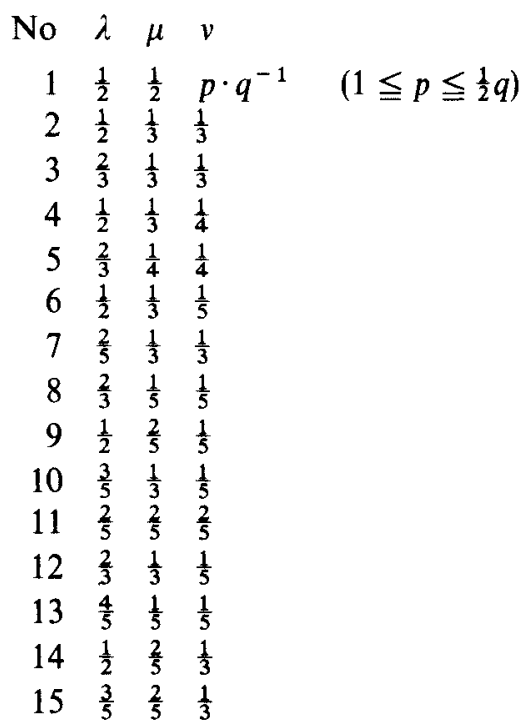


Theorem 5.5. Suppose $\boldsymbol{R}$ is an irreducible reduced root system with corresponding possibly non reduced root system

$$
R_{n r}=\{\alpha \in P ; \alpha \in R \text { or } 2 \alpha \in R\} .
$$

For $\lambda \in \mathfrak{h}^{*}$ and $k \in K$ a multiplicity function on $R_{n r}$ the hypergeometric function $F(\lambda, k ; z)$ associated with $R_{n r}$ is an algebraic function of its variables $z=\left(z_{1}, \ldots, z_{n}\right)$ if the reduced triples in the contiguity classes of

$$
\lambda_{\alpha}=2\left(\lambda, \alpha^{\vee}\right), \mu_{\alpha}=\frac{1}{2}-k_{\frac{1}{2} \alpha}-k_{\alpha}, v_{\alpha}=\frac{1}{2}+k_{\frac{1}{2} \alpha}+k_{\alpha}-2\left(\rho, \alpha^{\vee}\right)
$$

appear in the Schwarz list for all $\alpha \in R_{+}$.

Proof. This is just a reformulation of Corollary 4.10 taking into account Theorem 5.4 and formulas (4.5) of [HO]. Q.E.D.

Remark 5.6. For any root system $R$ we get algebraic hypergeometric function by taking $k_{\alpha}=0 \forall \alpha \in R_{n r}$ and $\lambda \in \mathfrak{b}^{*}$ rational with respect to the weight lattice $P$. The corresponding monodromy group is a semidirect product of $W$ and a finite factor group of $Q^{\vee}$ (namely $Q^{\vee}$ modulo $\left\{z \in Q^{\vee} ; w \lambda(x) \in \mathbf{Z} \forall w \in W\right\}$ ). This case is the higher dimensional analogue of No 1 in the Schwarz list.

Example 5.7. Suppose $R$ is of type $A_{2}$. We write $k_{\alpha}=k \forall \alpha \in R$, and $\lambda=n_{1} \lambda_{1}+n_{2} \lambda_{2}$. Then the condition of Theorem 5.5 implies that the three triples

$$
\begin{gathered}
\left\{2 n_{1}, \frac{1}{2}-k, \frac{1}{2}-k\right\} \\
\left\{2 n_{2}, \frac{1}{2}-k, \frac{1}{2}-k\right\} \\
\left\{2 n_{1}+2 n_{2}, \frac{1}{2}-k, \frac{1}{2}-3 k\right\}
\end{gathered}
$$

up to contiguity and order should appear in the Schwarz list. An easy verification using Theorem 5.4 yields the following possibilities besides the ones mentioned in Remark 5.6: $\left\{2 n_{1}=2 n_{2}=\frac{2}{3}, k= \pm \frac{1}{4}\right\},\left\{2 n_{1}=2 n_{2}=\frac{2}{3}, k= \pm \frac{3}{10}\right\}$.

Corollary 5.8. For $R$ an irreducible root system of type $A D E$ and rank $n \geqq 3$ there are no other algebraic hypergeometric functions than the ones described in Remark 5.6.

Proof. By rank reduction it is sufficient to verify this statement for type $A_{3}$, and the easy verification is left to the reader. Q.E.D.

\section{References}

[BH] Beukers, F., Heckman, G.J.: Monodromy for the hypergeometric function ${ }_{n} F_{n-1}$. Invent. Math. 95, 325-354 (1989)

[B] Bourbaki, N.: Groupes et algèbres de Lie, Ch 4,5 et 6. Paris: Masson (1981)

[B] Brieskorn, E.: Die Fundamentalgruppe des Raumes der regulären Orbits einer endlichen komplexen Spiegelungsgruppe. Invent. Math. 12, 57-61 (1971)

[D] Deligne, P.: Equations différentielles à points singuliers réguliers. (Lecture Notes Math., vol. 163) Berlin-Heidelberg-NewYork: Springer (1970)

[HO] Heckman, G.J., Opdam, E.M.: Root systems and hypergeometric functions, I. Compos. Math. 64, 329-352 (1987) 
[H1] Heckman, G.J.: Root systems and hypergeometric functions, II. Compos. Math. 64, 352-373 (1987)

[KL] Kazhdan, D., Lusztig, G.: Proof of the Deligne-Langlands conjecture for Hecke algebras. Invent. Math. 87, 153-215 (1987)

[vdL1] van der Lek, H.: Extended Artin groups. Proc. Symp. Pure Math. 40, Vol. 2, 117-122 (1981)

[vdL2] van der Lek, H.: The homotopy type of complex hyperplane complements. Thesis, Nijmegen 1983

[L1] Lusztig, G.: Some examples of square integrable representations of semisimple p-adic groups. Trans. Am. Math. Soc. 277, 623-653 (1983)

[L2] Lusztig, G.: Representations of affine Hecke algebras. Preprint MIT 1988

[N] Nguyên Viêt Dung: The fundamental groups of the spaces of regular orbits of the affine Weyl groups. Topology 22, 425-435 (1983)

[O1] Opdam, E.M.: Root systems and hypergeometric functions, III. Compos. Math. 67, 21-49 (1988)

[O2] Opdam, E.M.: Root systems and hypergeometric functions, IV. Compos. Math. 67, 191-209 (1988)

[O3] Opdam, E.M.: Some applications of hypergeometric shift operators. Invent. Math. 98, 1-18 (1989)

[S] Schwarz, H.A.: Über diejenigen Fälle in welchen die Gaussische hypergeometrische Reihe einer algebraische Funktion ihres vierten Elementes darstellt. Crelle J. 75, 292-335 (1873) 